Other factors predisposing to acute renal insufficiency induced by these drugs are advanced age, renovascular disease, systemic lupus erythematosus, and gout. All nonsteroidal anti-inflammatory drugs have been implicated in causing acute renal insufficiency, but indomethacin has been cited most commonly, perhaps owing to its greater use.$^{10}$ The drug sulindac has less effect on renal prostaglandin synthesis than other non-steroidal anti-inflammatory drugs," but it may nevertheless inhibit renal prostaglandin synthesis ${ }^{12}$ and cause renal failure. ${ }^{10}$ Fortunately this acute renal insufficiency is usually reversible on stopping the non-steroidal antiinflammatory drug.

The second form of renal toxicity that may be induced by non-steroidal anti-inflammatory drugs is hyperkalaemia. Prostaglandins directly stimulate the release of renin, and thus the inhibition of cyclo-oxygenase by non-steroidal antiinflammatory drugs leads directly to a hyporeninaemic hypoaldosteronism with subsequent hyperkalaemia. ${ }^{313}$ Stopping the drug quickly reverses the hyperkalaemia, but the serum potassium concentration may need to be monitored in patients treated with non-steroidal antiinflammatory drugs, particularly those having potassium supplements or potassium sparing diuretics.

Renal papillary necrosis is well recognised with analgesics such as phenacetin, ${ }^{14}$ but it may also result from long term use of non-steroidal anti-inflammatory drugs such as aspirin and indomethacin. ${ }^{9}$ Acute interstitial nephritis and the nephrotic syndrome are two further renal lesions that have been associated with the use of non-steroidal antiinflammatory drugs. ${ }^{15} 16$ The mechanism responsible here is uncertain-it may represent an allergy to the drug mediated through cytotoxic $T$ cells, ${ }^{15} 17$ and the mean duration of exposure to the drug before diagnosis has been six to eight months.' Renal biopsy specimens usually show a minimal change glomerular lesion with lymphocytic infiltration of the interstitium. Although many non-steroidal antiinflammatory drugs have been implicated in this condition there does seem to be an undue preponderance of cases associated with fenoprofen. ${ }^{79}$ In most cases the prognosis is good after stopping the drug, though treatment with steroids in high doses has been used to hasten recovery. ${ }^{17}$

As if this catalogue of renal disease induced by nonsteroidal anti-inflammatory drugs were not enough, Adams et al have added another problem. ${ }^{18}$ They reported that out of 17 patients presenting over three years with renal disease induced by these drugs, six had chronic renal failure. In contrast to the remaining 11 , the renal failure in these six patients did not improve when the drug was stopped. The lesion was papillary necrosis in one patient and interstitial fibrosis in the remaining five; this might possibly be the end stage of uncorrected interstitial nephritis. These authors suggested that the non-steroidal anti-inflammatory drugs with long plasma half lives were the ones most likely to cause this problem, but this remains speculation. ${ }^{18}$

What is clear is that many patients taking non-steroidal anti-inflammatory drugs are at risk of developing renal disease and that this risk is greatest in elderly patients; patients with cirrhosis, congestive heart failure, renovascular disease, or gout; and patients who are volume depleted or salt depleted. The largest group in this last category are those patients taking diuretics, whether loop diuretics such as frusemide or milder diuretics such as thiazides. Particular care needs to be taken when giving non-steroidal antiinflammatory drugs to patients taking diuretics, since many of these drugs inhibit the diuretic response to loop diuretics ${ }^{19}$ and the hypotensive response to thiazides ${ }^{20}$ in addition to their adverse effects on renal function.

M L'E ORME

Professor of Clinical Pharmacology,

University of Liverpool,

Liverpool L69 3BX

1 Ferreira SH, Moncada S, Vane JR. Indomethacin and aspirin abolish prostaglandin release from the spleen. Nature 1971;231:237-9.

Donker AJ, Arisz L, Brentiens JR, VanderHem GK, Hollemans HJ. Effect of indomethacin on kidney function and plasma renin activity in man. Nephron 1976:16:288-96.

Kidney RP. steroidal anti-inflammatory drugs. Am $\mathcal{F}$ Med 1978;64:804-7.

Lifschitz MD. Renal effects of non steroidal anti-inflammatory agents. $f$ Lab Clin Med 1983;102:313-23.

5 Speckart $\mathbf{P}, Z_{i a} P, Z_{i p s e r} R$, Horton $\mathbf{R}$. The effect of sodium restriction and prostaglandin inhibition on the renin-angiotensin system in man. $\mathcal{F}$ Clin Endocrinol Metab 1977; $44: 832-7$.

6 Levenson DJ, Simmons CE, Brenner BM. Arachidonic acid metabolism, prostaglandins and the kidney. Am $\mathcal{Y}$ Med 1982;72:354-74.

7 Stillman MT, Napier J, Blackshear JL. Adverse effects of non-steroidal anti-inflammatory drugs on the kidney. Med Clin N Am 1984;68:371-85.

8 Reeves WB, Foley RJ, Weinman EJ. Renal dysfunction from non-steroidal anti-inflammatory drugs. Arch Intern Med 1984;144:1943-4.

9 Carmichael J, Shankel, SW. Effects of non steroidal anti-inflammatory drugs on prostaglandins and renal function. Am 7 Med 1985;78:992-1000.

10 Blackshear JL, Davidman M, Stillman MT. Identification of risk for renal insufficiency from non teroidal anti-inflammatory drugs. Arch Inem Med 1983;143:11304.

11 Sedor JR, Williams SL, Chremos AN, Johnson CL, Dunn MJ. Effects of sulindac and indomethacin on renal prostaglandin synthesis. Clin Pharmacol Ther 1984;36:85-91.

12 Berg KJ, Talseth T. Acute renal effects of sulindac and indomethacin in chronic renal failure. Clin Pharmacol Ther 1985;37:447-52.

13 Tan SY, Shapiro R, Franco R, Stockard H, Mulrow PJ. Indomethacin induced prostaglandin inhibition with hyperkalaemia. Ann Intern Med 1979;90:783-5.

4 Prescott LF. Analgesic nephropathy - a reassessment of the role of phenacetin and other analgesics. Drugs 1982;23:75-149.

15 Bender WL, Whelton A, Beschorner WE, Darwish MO, Hall-Craggs M, Solez K. Interstitial nephritis; proteinuria and renal failure caused by non steroidal anti inflammatory drugs. Am $\mathcal{J}$ Med 1984;76:1006-12.

16 Brezin JH, Katz SM, Schwartz AB, Chinitz JL. Reversible renal failure and nephrotic syndrome associated with non steroidal anti inflammatory drugs. N Engl J Med 1979;301:1271-3.

17 Finkelstein A, Fraley DS, Stachura I, Feldman HA, Gandhy DR, Bourke E. Fenoprofen nephropathy; lipoid nephrosis and interstitial nephritis. A possible T lymphocyte disorder. Am f Med 1982;72:81-7.

18 Adams DH, Michael J, Bacon PA, Howie AJ, McConkey B, Adu D. Non-steroidal antiinflammatory drugs and renal failure. Lancet 1986; i:57-60.

19 Brater DC, Fox BS, Chennavasin P. Interaction studies with bumetanide and furosemide. Effects of probenecid and of indomethacin on response to bumetanide in man. 7 Clin Pharmacol 1981;21:647-53.

20 Favre $\mathrm{L}$, Glasson $\mathrm{Ph}$, Riondel $\mathrm{A}$, Valloton $\mathrm{MB}$. Interaction of diuretics and non-steroidal antiinflammatory drugs in man. Clin Sci 1983;64:407-15.

\section{Confidential inquiry into perioperative deaths}

A confidential inquiry into all perioperative deaths in three regions seemed impossible just over a year ago, but it started before Christmas in the South Western, North East Thames, and Northern regions. The inquiry will determine the incidence of particular causes of death and the part played by the anaesthetic, the surgery, and any other causes. Standards of care and clinical and organisational deficiencies will also be investigated. Funded by the Nuffield Provincial Hospitals Trust and King Edward's Hospital Fund for London, the inquiry is a joint effort between anaesthetists and surgeons-between the Association of Anaesthetists and the Association of Surgeons of Great Britain and Ireland.

After consultation with all the relevant clinical organisations, a working party prepared a protocol that received general approval. This scheme was then tested in Darlington, Exeter, and the Middlesex Hospital. A final questionnaire was then designed to show broadly what was done in surgical and anaesthetic management. It was intended neither as a detailed investigation nor to show the virtues of a particular approach but rather to describe the delivery of surgical and anaesthetic care in Britain today. 
Every death that occurs within 30 days of surgery in the three regions is being studied. Every hospital has agreed to take part after being visited by the clinical coordinators and having the study explained. In addition, just over $96 \%$ of the 1329 individual consultants are participating. The consultant surgeon and anaesthetist who were responsible for the case each receive a questionnaire, which is completed under the supervision of a consultant and returned to the office. Once all means of identification have been removed it is assessed. The assessors have been nominated by all the organisations initially consulted and include 275 surgeons from all subspecialties including gynaecology, 81 anaesthetists, and a range of other experts. The assessors comment, within their own specialties, on the events and on the standards of care.

More than 1300 deaths have been reported so far, and complete information has been collected on three quarters. The overall standards of care are good, but widely differing standards exist. The assessors have drawn attention to particular problems, which have been reported back at three private meetings to which participating consultants alone were invited. Firstly, both consultant surgeons and consultant anaesthetists are sometimes not participating in serious decisions that their trainees make about patients. Some registrars attempt surgery and anaesthesia far beyond their competence without guidance from their seniors. (One registrar has refused to complete a questionnaire because it is not included in his job description.)

A second problem is the quality of hospital notes. Some consultants have complained that their own notes are not adequate to complete the questionnaire: the weight of patients is often unrecorded, urine testing is overlooked, and prophylaxis against pulmonary thromboembolism and hepatorenal failure are commonly omitted even in patients who are known to be at particular risk. Thirdly, few deaths are reviewed collectively by the surgical and anaesthetic teams, and few districts have a regular mortality meeting or quality control mechanism. Coroners' necropsy findings are not routinely sent to the consultants, and one district has refused to pay the cost of obtaining photocopies of coroners' necropsy results for consultants.

Fourthly, most of the deaths are in elderly people, and many of the assessors have questioned the wisdom of operating on some of these old people. Furthermore, many of these elderly patients are subjected to long operations when they are in poor medical condition. That two or three days later they die of bronchopneumonia, cardiac failure, or electrolyte imbalance is hardly surprising since they already had these conditions before the operation, but the assessors have often expressed their profound disquiet at this sequence of events.

There are other examples that could be given, but they are at the moment only anecdotal illustrations of the type of information that will come from the study. We do not yet know how common they are because we do not know for certain the total numbers of operations. We estimate, however, that we have already studied deaths arising from 80000 to 100000 operations-so most patients do well.

The protocol of this exercise allows for its extension nationally in a form dependent on the outcome of the present inquiry. But whatever its form none of the clinical information is identifiable and all the numerical results will be pooled and published as regional data. Nobody need feel threatened: no personal identifying information can, or will, be released, and legal protection for the data is assured.

Feedback is, however, available. When the questionnaire is completed the doctor is asked to record a score for his team's management. He can, after the assessment is completed, contact the office, which, after identification and precautionary measures, will tell the inquirer the assessor's score of the management. No other information is divulged. Peer review is thus available for the asking, but so far a mere 20 doctors have asked.

H B Devi.in J N LUNN

Confidential Enquiry into Perioperative Deaths.

9 Bedford Square.

London WCIB 3RA

\section{The truth about the NHS?}

The NHS is becoming a political, statistical, and managerial battleground. The Conservative government claims that more doctors and more nurses are spending more money in treating more patients than when it entered office seven years ago. Indeed, a press release on Norman Fowler's recent address to regional health authority chairmen was titled "The NHS - seven years of progress." Many doctors, nurses, and other NHS staff would dispute the Secretary of State's confident diagnosis. Yet his statistics sound optimistic enough. In 1986 the United Kingdom will be spending $£ 18 \cdot 75 \mathrm{~m}$ on the NHS, $£ 340$ for every man, woman, and child in the country compared with $£ 140$ spent in 1978-9.

With almost 500000 nurses and around 50000 hospital doctors and dentists there are eight nurses now for every seven in 1978 and nine doctors for every eight, with 3500 more general practitioners and 3000 more general dental practitioners. These staff treated nearly 1 million more inpatient cases and 400000 more day cases and dealt with 3.75 million more outpatient attendances. The number of patients receiving kidney treatment has doubled, three times as many coronary bypass grafts are being done, and seven total hip replacements are being completed for every five in 1978. In 1986 over $£ 950 \mathrm{~m}$ is being provided for the capital programme in which 159 major projects are now being planned, designed, or built. "This," claims Mr Fowler, "is a developing health service not one in decline." And he even remembered to discuss quality of care as well as "better management," "cost improvements," and "competitive tendering."

Given that apparently roseate scene, why is there obvious discontent and low morale in the service, why is the media so consistently critical, why did the BMA, the Royal College of Nursing, and the Institute of Health Services Management present such a damaging critique of the NHS's finances earlier this year,' and why has the parliamentary opposition seized on health as a major front for attack in the run up to the election? Succinctly, what is the truth about the NHS?

The answers lie in the massive complexity of the NHS, the largest organisation in Europe outside the Red Army. This unwieldy organisation is responsible for one of the few growth areas in the British economy, and the demands of health care look set to keep on growing. Indeed the rate of growth in the "high tech" specialties such as radiology, cardiology, and orthopaedics has been faster than the service could afford-and as a result "ordinary" acute services and the "low tech" disciplines such as psychiatry and long stay care for the chronic sick have been cut, beds closed, and services reduced. However unpalatable this truth may be to 